Earth had initially formed and the core had become distinct from the mantle. So, according to these results, the gold in your jewellery can be viewed as a relative latecomer to our planet.

The debate about the highly siderophile elements has centred on why they are as abundant in the mantle as they are. The evidence for thinking that their abundance should be much lower comes from chondritic meteorites. These are bits and pieces left over from the formation of the early Solar System that have fallen to Earth, and are the most primitive materials available for comparison. Chondritic meteorites have abundances of highly siderophile elements of between 150 and 300 times that of the mantle, yet the mantle concentrations predicted by metal-silicate magma partitioning experiments conducted at 1 atmosphere of pressure are more than two orders of magnitude lower ${ }^{2}$. That the concentrations are not as low as predicted is fortuitous for jewellers (and others), because viable economic deposits of these elements would probably not have formed from a mantle with the much lower concentrations. One other curious aspect about the mantle abundances of the highly siderophile elements is that, on a coarse scale at least, they occur in 'chondritic relative abundances' meaning that the ratio of the abundance of one element to another in the mantle is similar to the ratio in chondritic meteorites ${ }^{3}$.

Over the past 40 years, many models have been proposed to explain the abundances of these elements in the mantle. But recent discussion has largely settled on two possibilities. In one, it has been proposed that metal-silicate magma segregation may have occurred during the first 100 million years of the Earth's history at the base of a deep magma ocean (extending to $500-1,000 \mathrm{~km}$ beneath the surface), and that the affinity of these elements for iron may decrease as a function of pressure, thereby better matching the observed concentrations ${ }^{4-6}$.

A flurry of high-pressure partitioning experiments during the past decade has shown that this model is robust for at least explaining the abundances of some lesssiderophile elements, such as nickel and cobalt ${ }^{5}$. The main constraint to this model is that a condition has to be found under which all of the highly siderophile elements have a low enough affinity for metallic iron to account for their abundance in the mantle. Those affinities must be nearly the same to account for the chondritic relative abundances. One study ${ }^{6}$ has reported that rhenium exhibits a greatly reduced affinity for iron at higher pressures, lending credence to this model. Until the work of Holzheid and colleagues ${ }^{1}$, however, there have been few data for other highly siderophile elements.

The second possibility has been termed the 'late veneer' or 'heterogeneous accretion' model. This attempts to explain the mantle abundances of the highly siderophile elements by appealing to the addition of $0.5-1 \%$ of oxidized chondritic material to the Earth after the core had formed ${ }^{7,8}$. The relatively high abundances of these elements in chondrites would dramatically raise their concentrations in a primitive mantle that had effectively been stripped of them by core formation. The oxidized nature of the material would not allow the highly siderophile elements to move back into the core.

Holzheid et al. ${ }^{1}$ describe results for the partitioning of platinum and palladium between metal and silicate magmas at high pressures (equivalent to depths of $500 \mathrm{~km}$ ) and temperatures. Such experiments are notoriously difficult. In part this is because of the low concentrations of the elements present in silicate magma equilibrated with metal magma. More troublesome, however, is the tendency for tiny nuggets of the elements to form and not segregate readily from the silicate into the metal magma. This can lead to seemingly higher concentrations of the element in the silicate than is consistent with equilibrium partitioning.

For the latest experiments ${ }^{1}$, palladium was chosen because of its limited tendency to form nuggets. Nugget formation is still an issue with platinum, but the study at least provides minimum constraints on its affinity for metallic iron. The results show that both palladium and platinum retain very high affinities for metal relative to silicate magma under high pressures and temperatures, and that, at least for the conditions tested, the process could not lead to the abundances of these two elements found in the mantle.

So the results cast doubt on the ability of the high-pressure metal-silicate segregation model to account for the mantle abundances of highly siderophile elements, and are consistent with the late-veneer model. They do not provide evidence against the purported deep segregation of metal and silicate magmas, however. Ultimately it may transpire that some elements in the mantle, such as the less-siderophile nickel and cobalt, owe their abundances to metal segregation at the base of a deep magma ocean, and that only the highly siderophile elements are dominated by the late-veneer signature?

Richard J. Walker is in the Department of Geology, University of Maryland, College Park, Maryland

20742, USA.

e-mail: rjwalker@geol.umd.edu

1. Holzheid, A., Sylvester, P., O’Neill, H. St C., Rubie, D. C. \&

Palme, H. Nature 406, 396-399 (2000).

Jones, J. H. \& Drake, M. J. Nature 322, 221-228 (1986).

Chou, C.-L., Shaw, D. M. \& Crocket, J. H. J. Geophys. Res. 88, A507-A518 (1983)

4. Murthy, R. Science 253, 303-306 (1991)

Li, J. \& Agee, C. B. Nature 381, 686-689 (1996).

6. Righter, K. \& Drake, M. J. Earth Planet. Sci. Lett. 146, 541-553 (1997)

Chou, C.-L. Proc. Ninth Lunar Planet. Sci. Conf. 219-230 (Pergamon, New York, 1978)

8. Morgan, J. W. J. Geophys. Res. 91, 12375-12387 (1986)

9. Drake, M. J. Geochim. Cosmochim. Acta 64, 2363-2370 (2000).
Daedalus

\section{Hidden thoughts}

Last week Daedalus proposed a method of measuring brain activity, in neural pulses per second. Each pulse puts the sodium ions of the relevant nerve into brief 1-kilohertz oscillation. At that moment, their nuclear magnetic excitation at $1 \mathrm{kHz}$ is strongly damped. DREADCO volunteers are now testing a nuclear magnetic psychometric hat, which measures the wearer's changing sodiumion relaxation density, locates the sources of this activity by magnetic imaging, and transmits the results to a base station.

The steady cerebral base load of bodily 'housekeeping', keeping the heart and lungs pumping, and so on, should be fairly constant. Processing due to perception and conscious thought will vary wildly, but in keeping with the subject's circumstances. Sensory processing will be identified by its decline during sensory deprivation, and conscious thought by standard tasks and tests. The most interesting study will be subconscious thought. Even psychologists find it an elusive concept.

Daedalus feels that the subconscious mind cares little for the conscious needs of the moment. Thus a creative scientist can have a new idea at any time. The subconscious may have been developing the idea for ages before suddenly 'pushing it upstairs' for conscious consideration. With luck, one of Daedalus's volunteers will have such an inspiration while wearing the psychometric hat, thus capturing the whole sequence. It should show the subconscious activity, the burst of inspiration, and the transfer of activity to a different region as the conscious mind considers the new idea.

The subconscious also handles personal problems. Volunteers wrestling with superego-id conflicts, or shouldering or rejecting guilt or anger, or arguing with internalized parent figures, should reveal the fact by extra heavy processing in specific regions.

Once identified, subconscious activity could be tamed and trained by biofeedback methods. Shown their brain activity in real time, the volunteers could learn how to control it. Chronic worriers will soothe away their fears; those sabotaged by repressed memories or fatigued by obsessive notions will learn, if not to damp them out, at least to run them efficiently with the minimum number of neurons. Even conscious thought could be neurologically streamlined. The mental space thus opened will give a new freedom and spaciousness to mental life, and make room for more creative imagination. David Jones 